\title{
Importance of thymectomy and prognostic factors in the complex treatment of myasthenia gravis
}

\author{
Bak V ${ }^{1}$, Spalek $\mathrm{P}^{2}$, Rajcok $\mathrm{M}^{1}$, Danihel L ${ }^{1}$, Schnorrer $\mathrm{M}^{1}$ \\ 3rd Department of Surgery of University Hospital Bratislava, Bratislava, Slovakia. mrvbak@gmail.com
}

\begin{abstract}
BACKGROUND: Thymectomy is indicated in patients with seropositive myasthenia gravis (SPMG) in patients under the age of 50 and in patient with myasthenia gravis (MG) associated with thymoma.

METHODS: 345 patients with MG who underwent an extended thymectomy from April 1990 to December 2010. Patients were separated into the 4 groups: group with a complete stable remission, pharmacological remission group, group of patients with significant improvement and the group with mild improvement of symptoms. RESULTS: In our study, we observed 345 patients with MG and thymectomy. 137 patients $(39.71 \%)$ attained the complete stable remission (CSR) and 92 patients $(26.67 \%)$ the pharmacological remission (PR). The significant improvement of MG symptomatology was achieved in 95 patients $(27.54 \%)$. The rest of 21 patients from total $345(6.08 \%)$ have reached only a mild improvement. Patients after thymectomy with CSR were in the clinical stage I and IIA, in accordance to the modified Osserman classification and most benefited from the thymectomy. CONCLUSIONS: The extended thymectomy combined with immunotherapy is the preferred treatment with an expected satisfactory long-term remission rate. Despite the recent improvements in MG medical therapy, thymectomy plays an important role in the otherwise complex treatment of the disease. Because of early diagnosis with thymectomy performed without a delay, patients can achieve significantly more often favorable outcomes and even stable remissions (Tab. 4, Fig. 7, Ref. 17). Text in PDF www.elis.sk. KEY WORDS: myasthenia gravis, thymoma, thymectomy, outcome, prognostic factors.
\end{abstract}

\begin{abstract}
Abbreviations: MG - myasthenia gravis; EOMG - early onset of myasthenia gravis; LOMG - long onset of myasthenia gravis; SPMG - seropositive myasthenia gravis; SNMG - seronegative myasthenia gravis; MuSK - muscle specific kinase; MGFA - Myasthenia Gravis Foundation of America; MGAT - myasthenia gravis assosiated with thymoma; CSR - complete stable remission; PR - pharmacological remission; SI - significant improvement; MI - mild improvement.
\end{abstract}

\section{Introduction}

The role of thymus in the complex pathogenesis of myasthenia gravis $(\mathrm{MG})$ has been largely clarified $(1,2)$. Thymectomy contributes significantly to the treatment of seropositive myasthenia gravis (SPMG) in patients under the age of 50. Immunocompetent structures, as well as myoid cells, which have acetylcholine receptors on their surface and are the site of initiation of the autoimmune pro-

13rd Department of Surgery of University Hospital Bratislava, Slovakia, and ${ }^{2}$ Centre for Neuromuscular disorders of Neurological clinic, University Hospital Bratislava - Ruzinov, Bratislava, Slovakia

Address for correspondence: V. Bak, MD, 3rd Department of Surgery of University Hospital Bratislava, SNP 10, SK-814 65 Bratislava, Slovakia. Phone: +421.2 .57887400$

Acknowledgement: We are grateful to Paul Janotka for the language revision of the manuscript. The submitted work was performed only with the funding of the national health system. cess, are removed by thymectomy. Thymectomy has an insufficient effect on SPMG in about $25-30 \%$ of patients and after the surgery the long-term immunosuppressive therapy is usually needed $(3,4$, $5,6)$. Thymectomy is not indicated in patients with SPMG over the age of 50. In this group of patients, the pathological finding is primarily atrophy of the thymus $(2,7,8)$. From the surgical and oncologic point of view, the presence of a thymoma is the absolute indication for thymectomy. But the operation has quite often an insufficient effect on $\mathrm{MG}(5,9,10)$. Anyway, thymectomy is ineffective in patients with seronegative myasthenia gravis (SNMG) with autoantibodies against muscle-specific kinase (MuSK). Currently, this form of MG is not indicated for thymectomy (11).

One of the most important prognostic factors is the duration of $\mathrm{MG}$ from the origin to the initiation of the treatment (with the exception of acute fulminant forms of MG) $(2,3,6)$. The range of irreversible structural changes of the postsynaptic plate and $\mathrm{ACh}$ receptors is increasing with the duration of $\mathrm{MG}$ and their natural regenerative abilities are being limited at the same time. Therefore, in the insufficiently effective or the late treatment, the chance for the complete cure or for the substantial clinical state improvement by comprehensive treatment is significantly smaller than in the initial stages of the disease. Moreover, poorly treated or untreated MG usually progresses and may result in myasthenic crisis. For MG is also true that even after a long period of clinical or pharmacological remission there are possible exacerbations of the disease at myasthenics. 


\section{5-200}

There are still ongoing discussion about the proper use of thymectomy regarding to outcome and benefit in various forms of MG. Radical operation with removal of the ectopic thymic tissue has shown to be the most positive and significant prognostic factor for treatment of MG (12). The aim of this article is to highlight 20 years of experience and the results of a comprehensive treatment of operated patients with MG as well as to determine prognostic factors that may potentially influence the overall outcome of MG patient.

\section{Materials and methods}

In the retrospective review, we evaluated the results of the comprehensive treatment of patients with MG who underwent surgery (thymectomy). After diagnosis and evaluation by neurologist before the surgery, the treatment plan was determined and medications were initiated. The treatment led to the improvement of MG symptoms before the surgery. The majority of patients received immunosuppressive therapy/prednisone and azathioprine/ before the surgery. Combination of plasmaphoresis and intravenous immunoglobulin were employed in some cases in addition to immunosuppressives. When a desired decrease of clinical symptoms of MG was achieved, the low-dose immunosuppressive maintenance therapy was initiated in spite of the fact that most of the patients were asymptomatic that time. Only after the abovementioned complex medical preoperative treatment, the surgical procedure was performed. The preoperative drug therapy was necessary to improve tolerance to general anaesthesia in order to make the surgical procedure safer. MG symptoms and expected complications were kept under control as much as possible before the planned surgery.

369 patients underwent surgery in connection with the treatment of MG between April 1990 and December 2010. We excluded 24 of them due to incomplete clinical data. The baseline characteristics of patients are shown in Table 1. The mean follow up time of 345 operated patients was 9.9 years (min. 2.0 - max. 20.0 years).

In this study, we tried to identify and assess factors such as gender, age, mean time of history of illness, mean time of the lengths of preoperative treatment, mean time from the point when diagnosis was made to the time of surgery. Other factors were also assessed, such as histopathology findings associated with given MG patient and clinical stage of given MG patient according to

Tab. 1. Baseline characteristics of patients.

\begin{tabular}{lc}
\hline Characteristics & $\mathrm{n}=345$ \\
\hline $\begin{array}{l}\text { Sex }- \\
\text { male / female }\end{array}$ & $81 / 264 \sim 23.48 \% / 76.52 \%$ \\
\hline $\begin{array}{l}\text { Age - Median } \\
\text { (min. - max.), years }\end{array}$ & 33 (min. $18-$ max. 68) \\
\hline $\begin{array}{l}\text { Average time of history of present } \\
\text { illness to diagnosis (months) }\end{array}$ & $9.3 \pm 4.46$ \\
\hline $\begin{array}{l}\text { Mean time of preoperative } \\
\text { treatment (months) }\end{array}$ & $10.70 \pm 7.26$ \\
\hline $\begin{array}{l}\text { Mean time of history and } \\
\text { preoperative treatment (months) }\end{array}$ & $7.69 \pm 8.36$ \\
$\begin{array}{l}\text { Mean time of staying at hospital } \\
\text { after thymectomy (days) }\end{array}$ & $7.05 \pm 4.3$ \\
\hline
\end{tabular}

modified Osserman's classification. The influence of those factors on development, course of disease and treatment of MG were analyzed. We also evaluated the effect of radical thymectomy to overall improvement of MG symptoms and the occurrence of postoperative complications of this procedure. Histopathology findings of thymic tissue as well as type and stage of thymoma were significant factors to select patients for the certain distinguished group for the sake of having the most objective analysis of the available data.

Upon the completion of the surgery, all patients were followed up by a neurologist in the Centre for treatment of MG and other neuromuscular disorders. Different outcomes of MG treatment in the sense of clinical improvement and remission of symptoms have been achieved. Based on those differences, patients were separated into the 4 groups. The first group of patients achieved a clinical stable remission (CSR) and they needed no additional treatment. In the second group, we included asymptomatic patients in the pharmacological remission (PR). They required the maintenance immunosuppressive therapy without corticosteroids. Third group included patients with significant improvement (SI) in the sense of a substantial reduction of clinical signs and symptoms of MG when compared to the clinical state before thymectomy. These patients also required constant immunosuppressive therapy. Fourth group consisted of patients with persistent MG symptoms in spite of thymectomy and immunosuppressives. They tolerated normal daily activities well, but they were poorly tolerant to various degrees of physical stress.

The success of the complex treatment of patients with $\mathrm{MG}$ is measured by the achievement of CSR and PR. Adjuvant oncologic therapy was initiated by an oncologist in the case of positive histopathology for malignancy - malignant thymic tumours. Histopathological findings were originally classified according to a former morphological system proposed by Bernatz and then by Muller-Hermelink. We had tried to use the most recent WHO classification system. Due to not unified histopathology classification in the past 20 years, we had been not able to distribute patients according to the newest classification system. Therefore, we divided the patients into the two groups based on 2 histological types: thymoma or thymic carcinoma.

Our preferred surgical access for thymectomy was and still is the upper partial sternotomy. It provides a good and safe exposure of upper anterior mediastinum. After 10 to $12 \mathrm{~cm}$ skin incision, with the sternum retracted, adequate visualization of thymus and its cervical extensions is obtained to execute a safe total radical thymectomy. Whole thymus and adjacent fatty tissue from anterior mediastinum were removed. Once the thymus was dissected and separated completely from adjacent structures, the pericardium, the ascending aorta, the anonymous vein and both pleural surfaces were visualized. The sternum was then sutured using three metal wires. A chest tube was placed retrosternaly for an adequate postoperative drainage of the mediastinum.

For the proper data description, data analysis and presentation, frequency tables were posted. In this effort, we used basic data characteristics such as the mean time, mean age, median and range. To present different treatment achievements in the treatment of MG, our study data were calculated as the percentage from total. 
The Pearson Chi-Square and the Fisher exact test were used to analyze the difference between the studied variables. Groups of patients sorted by the mentioned criteria were evaluated with the Kaplan-Meier method. The differences in survival curves were tested with Mantel-Cox test and generalized Wilcoxon test. The efficiency of the radical thymectomy was assessed. Obtained results are based on the numeric count of the complete resections in the different stages of detected tumours classified by Masaoka.

\section{Results}

There were total 345 patients in our study. 137 patients (39.71\%) of them attained a complete stable remission. 92 patients (26.67\%) from total achieved a pharmacological remission. The success in the complete stable and pharmacological remission has reached 229 patients $(66.38 \%)$. The significant improvement of MG symptoms had been achieved in 95 patients (27.54\%). The rest of 21 patients $(6.08 \%)$ have reached only a mild improvement.

The impact of the disease duration (from the beginning of symptoms until the time of diagnosis) and its influence to overall outcome was observed and made a significant difference. The outcome of treatment was also affected by the mean time of the preoperative drug therapy and by the total duration of the disease up to the date of the surgery. It is clear and provable that early diagnosis of MG and a shorter time of the preoperative therapy have beneficial effect in achieving the remission. The figure 1 presents the time values in month: different time duration of the illness in different groups of patients. Results significantly differs using the Kruskal-Wallis statistical analysis. $(p=0.048 ; p=0.47 ; p=0.036)$.

In accordance with the modified Osserman's classification (Tab. 2), the most significant benefit is expected in patients after thymectomy with determined stage I and IIA. The complete stable

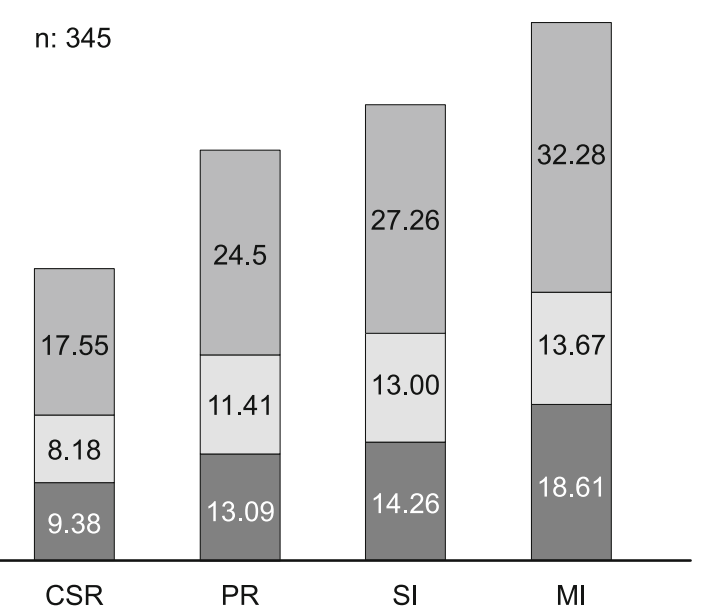

Mean time of history and preoperative treatment

Mean time of preoperative treatment

Mean time of history of present illness

Fig. 1. Relation between the mean time of history of present illness and preoperative treatment related to the outcomes of complex treatment.
Tab. 2. Patients' distribution in pathogenic form of MG and results of the complex treatment.

\begin{tabular}{lcccc}
\hline $\begin{array}{l}\text { Clinical stage } \\
\text { of MG }\end{array}$ & CSR & PR & SI & MI \\
\hline I (n:13) & $8 \sim 61.5 \%$ & $5 \sim 38.5 \%$ & $0 \sim 0.0 \%$ & $0 \sim 0.0 \%$ \\
IIA (n:100) & $47 \sim 47.0 \%$ & $27 \sim 27.0 \%$ & $21 \sim 21.0 \%$ & $5 \sim 5.0 \%$ \\
IIB (n:183) & $57 \sim 31.3 \%$ & $71 \sim 39.0 \%$ & $52 \sim 28.6 \%$ & $2 \sim 1.1 \%$ \\
III (n:49) & $3 \sim 6.0 \%$ & $14 \sim 28.0 \%$ & $12 \sim 24.0 \%$ & $20 \sim 40.0 \%$ \\
\hline
\end{tabular}

Tab. 3. Patient's distribution in clinical stage of MG and results of the complex treatment.

\begin{tabular}{lcccc}
\hline $\begin{array}{l}\text { Pathogenic } \\
\text { form of MG }\end{array}$ & CSR & FR & VZ & MZ \\
\hline SPMG (n:200) & $88 \sim 43.7 \%$ & $55 \sim 27.3 \%$ & $51 \sim 25.4 \%$ & $6 \sim 2.9 \%$ \\
\hline SNMG (n:66) & $21 \sim 31.8 \%$ & $22 \sim 33.3 \%$ & $20 \sim 30.3 \%$ & $3 \sim 4.5 \%$ \\
\hline MGAT (n:47) & $2 \sim 4.2 \%$ & $11 \sim 23.4 \%$ & $22 \sim 46.8 \%$ & $12 \sim 25.5 \%$ \\
\hline $\begin{array}{l}\text { Unknown form } \\
\text { of MG (n:32) } \\
\text { (incomplete data) }\end{array}$ & 26 & 4 & 2 & 0 \\
\hline
\end{tabular}

Tab. 4. Patients' distribution with different histological findings and results of the complex treatment.

\begin{tabular}{lcccc}
\hline Histological findings & CSR & PR & SI & MI \\
\hline Hyperplasia (n:166) & $94 \sim 56.6 \%$ & $33 \sim 19.9 \%$ & $34 \sim 20.5 \%$ & $5 \sim 3.0 \%$ \\
Atrophy (n:132) & $41 \sim 29.9 \%$ & $48 \sim 34.8 \%$ & $39 \sim 28.3 \%$ & $4 \sim 2.9 \%$ \\
Thymoma (n:47) & $2 \sim 4.3 \%$ & $11 \sim 23.4 \%$ & $22 \sim 46.8 \%$ & $12 \sim 25.5 \%$ \\
\hline
\end{tabular}

remission is frequently achieved in patients classified in this stages (Pearson Chi-Square $\mathrm{p}=0.01$ ). However, the clinical stage had no significant impact on the achievement of pharmacological remission while patients with mild symptomatic improvement were mostly assigned to the MG clinical stage III.

$60.27 \%$ of all patients with clinical remission carried seropositive form of MG. On the other hand, all patients with seropositive form of MG represented the highest proportion of patients that achieved the complete clinical remission (Tab. 3) (Pearson ChiSquare $\mathrm{p}=0.000$; Fisher-exact test $\mathrm{p}=0.000$ ).

The most cases of diagnosed SPMG correlated with the postoperative histopathological result of the thymic hyperplasia. Patients with the complete stable remission represented $68.61 \%$ (n $=94$ ) of all histopathological cases positive for thymic hyperplasia. Simultaneously, the highest portion of patients (56.6\%), who achieved the complete clinical remission, was in the group of patients with the thymic hyperplasia (Tab. 4). Significant and mild clinical improvement was achieved mostly in patients with histopathological findings of thymoma (Pearson Chi-Square $\mathrm{p}=$ 0.000 ; Fisher-exact test $p=0.000$ ). There was no statistical difference between histologically benign and malignant tumors. We found no significant statistical difference with regard to the outcome between this two subsets of patients (Pearson Chi-Square $p$ $=0.315$; Fisher-exact test $\mathrm{p}=0.376$ ).

The complete resection of tumours was performed in 43 (92\%) cases with stages I, II, III and only in 4 ( $8 \%$ ) patients with stage III and IVa. In the cases of the advance stage of thymic carcinoma with infiltration of great vessels and pericardium, a limited subtotal resection (debulking) of tumour was performed. However, the 


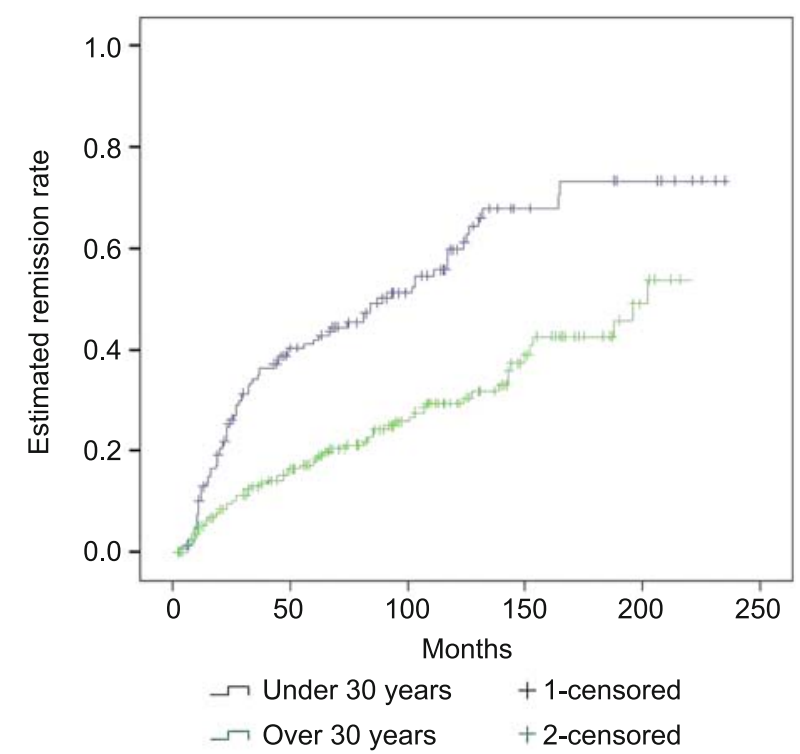

Fig. 2. The Impact of age on the complete stable remission; (n: 345); (Mantel-Coxov log-rank test $\mathbf{p}=\mathbf{0 . 0 0 0}$ )

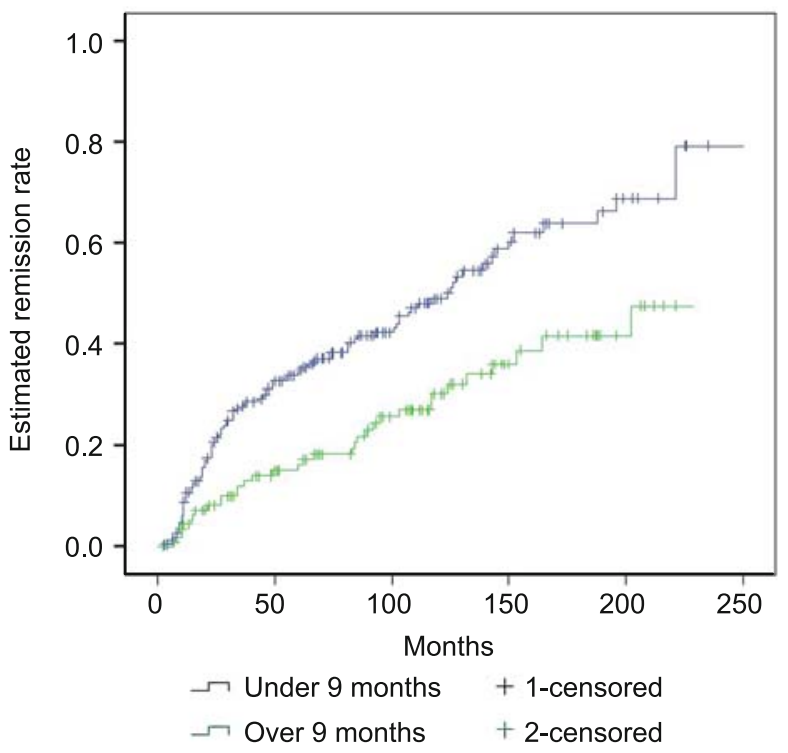

Fig. 3. The impact of symptoms duration based on patient history on the complete stable remission; (n: 345); (Mantel-Coxov log-rank test $\mathbf{p}=\mathbf{0 . 0 0 0}$ )

stage III thymic tumours were completely resected only in $57.1 \%$ of patients. The rest of the patients $(16.7 \%)$ diagnosed with stage III and IVa received an adjuvant radiotherapy.

Statistical analysis by the Kaplan-Meier method and MantelCox log-rank test was focused on the evaluation of prognostic factors, which could influence the accomplishment of the complete MG clinical remission. We found that patients after thymectomy under the age of 30 could achieve substantially better clinical results $(p=0.000)$ (Fig. 2). Important prognostic factors are the duration of symptoms based on historical data provided by the pa-

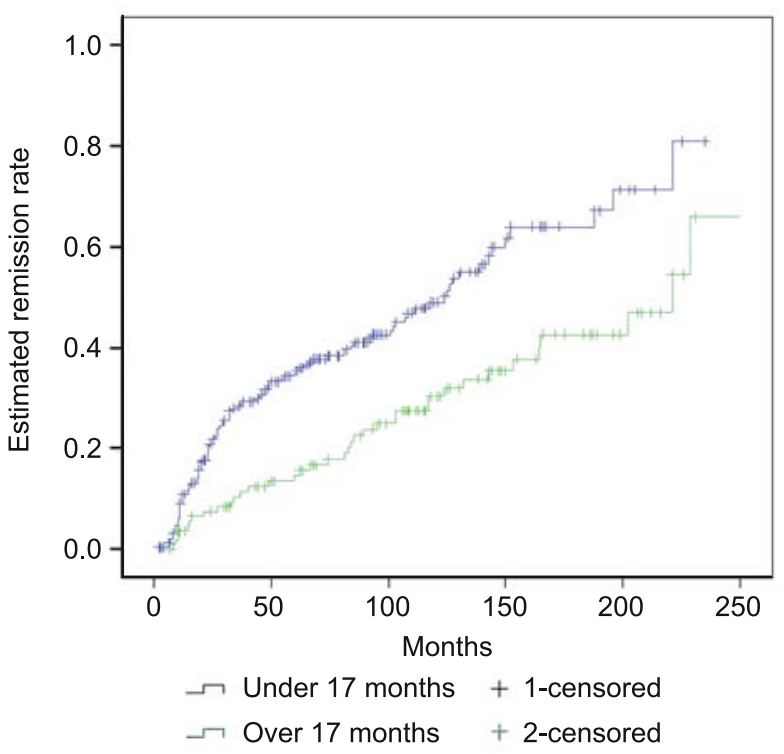

Fig. 4. The impact of the duration of disease up to surgery on the complete stable remission; ( $\mathrm{n}$ : 345$)($ Mantel-Coxov log-rank test $\mathrm{p}=\mathbf{0 . 0 0 0})$

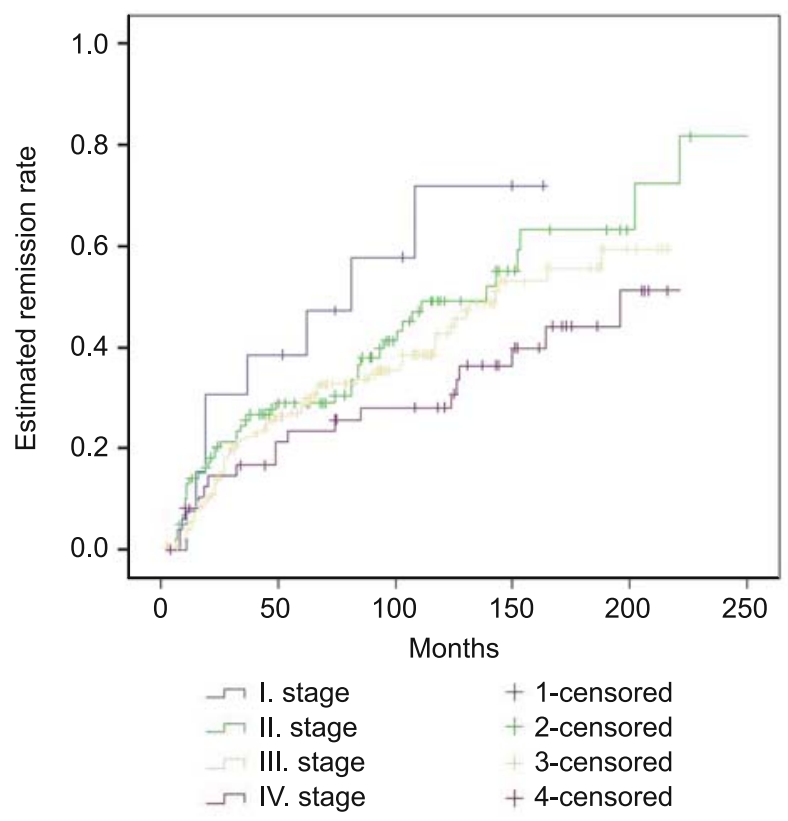

Fig. 5. The impact of clinical stage on the complete stable remission; (n: 345) (Mantel-Coxov log-rank test $\mathbf{p}=\mathbf{0 . 0 0 0})$

tient and the total duration of MG symptoms up to the surgery. The study showed that if the duration of symptoms (historical data) was within 9 months from the beginning (Fig. 3) and if the total duration of the disease was within 17 months until the surgery, there was much higher probability to achieve favourable outcomes and even CSR (Fig. 4). It shows that early diagnosis of the disease with the effective preoperative preparation and subsequent early surgery had the beneficial effect on the overall outcomes. Generalization of disease determined by clinical stage of MG negatively affects 


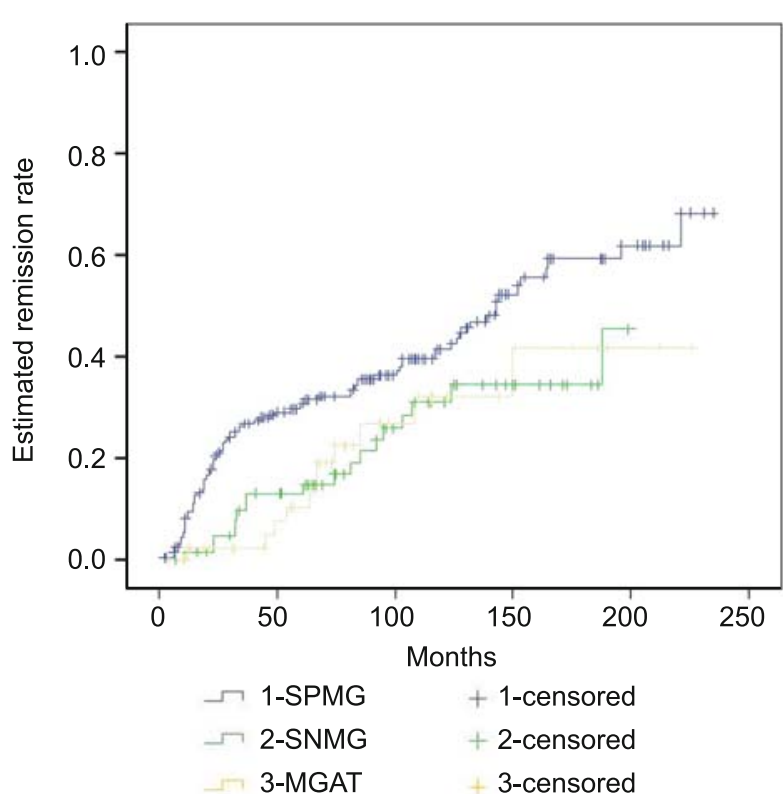

Fig. 6. The impact of pathogenic forms on the complete stable remission; (n: 345) (Mantel-Coxov log-rank test)

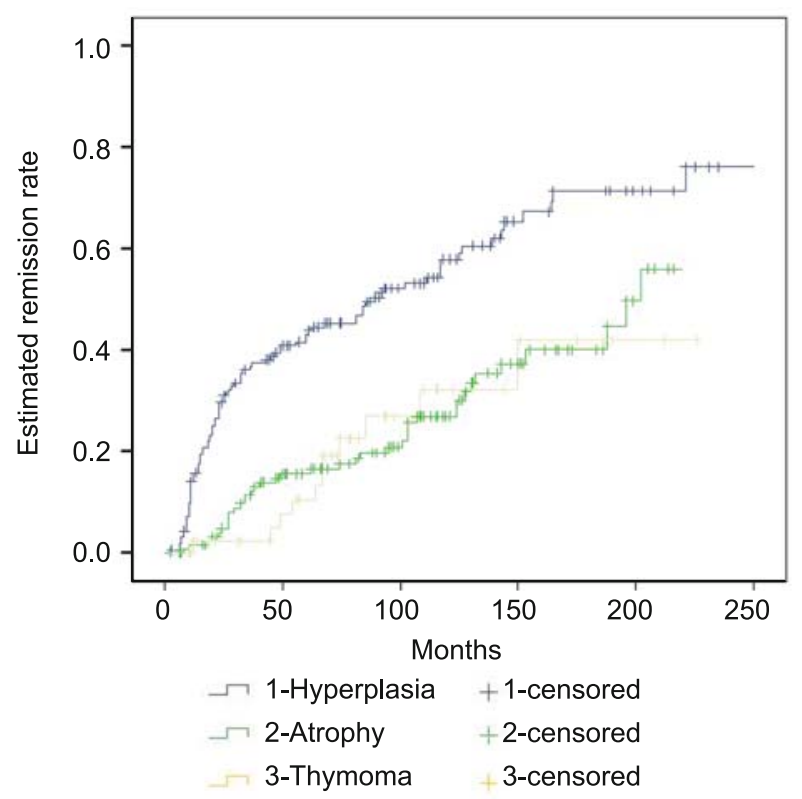

Fig. 7. The impact of histological findings on the complete stable remission; (n: 345) (Mantel-Coxov log-rank test $\mathbf{p}=\mathbf{0 . 0 0 0}$ )

the chance to achieve the remission. All patients with the ocular form of $\mathrm{MG}$ achieved a remission and a regression of symptoms (Fig. 5). Significant remission was achieved in a shorter time in this group compared to the patients with generalized form of $\mathrm{MG}$ $(p=0.000)$. At the same time, the presence of seropositive forms of $\mathrm{MG}(\mathrm{p}=0.000)$ (Fig. 6) and the presence of thymic hyperplasia were favourable prognostic factors $(p=0.000)$ (Fig. 7). Conversely, the finding of thymoma was the adverse prognostic factor for the clinical remission.
There was one case of death in our 20-years' surgical experience of performing radical transternal thymectomy. Thymectomy was performed as the last option after exhausting all conservative treatment available for a given patient in the case of myasthenic crisis to ameliorate clinical condition. There were recorded 29 cases of minor complications in the postoperative period that were managed conservatively. Any other consequential complications were not observed, which set the postoperative morbidity to $9.5 \%$.

\section{Discussion}

No prospective and randomized study until present has clearly confirmed the effectiveness and the role of thymectomy in the treatment of MG. Nevertheless, the thymectomy is still regarded as an effective mean in helping to reduce and eventually completely eliminate the need for medication therapy. The early performed thymectomy is one of the important steps in the complex management and treatment of the MG cases (13). The equivocal answer to the question of the effectiveness of thymectomy in present time could bring an unequivocal one in the year 2015, when ongoing study, initiated by John Newson-Davies, will be platted. The study is comparing end results of thymectomy with corticosteroid and corticosteroid therapy alone (14).

The significant changes in the attitude toward surgical treatment resulted in early indications for procedure and much better surgical outcomes with low mortality and morbidity rate. Further progresses in understanding of MG pathogenesis and immunology aspects of disease led to focused and targeted immunosuppressive preoperative therapy, which made the thymectomy as operative intervention even safer. There is an ongoing debate about the adequate extent of thymectomy. Numerous studies demonstrated the importance of the removal of mediastinal ectopic thymic tissue. Extended transternal thymectomy using the partial sternotomy approach offers good exposure of the anterior mediastinum for safe and complete removal of the thymus as well as the removal of pre-tracheal parathymic mediastinal and cervical fat tissue, where the ectopic thymic tissue is mostly and frequently located (13).

Employing the complex treatment of operated patients with $\mathrm{MG}$, the both complete stable and pharmacological remission were achieved in $66 \%$. Patients in clinical remission were asymptomatic without the medication. Patients in pharmacological remission required the low-dose maintenance immunosuppressant therapy with no corticosteroids added. Patients who experienced the exacerbation of MG symptoms were managed by medical therapy and a pharmacological remission was achieved. The extended transternal thymectomy is the effective operative option for the complete removal of thymus and pre-tracheal parathymic tissue to set favourable stage for stable remission of MG symptoms. According to our experience, the partial sternotomy provides sufficient and safe access to anterior mediastinum.

Statistical analysis found several important prognostic factors, which influenced the clinical and pharmacological remission. Analyzing the set of our patients based on their mean age with regard to their clinical remission, we found that the beneficial prognostic factor was the age below 30 . The most likely cause for 
different outcomes in MG treatment was an early indication for the surgery, which led to decreased comorbidity and better tolerance to aggressive medication treatment and by then better chance to tolerate higher doses of drugs.

The seropositive form of MG, which formation is conditioned by the presence of myeloid cells, is beneficial prognostic factor with the statistically significant prevalence. Conversely, the presence of thymoma, which is the absolute indication for surgery, is the adverse factor for desired remission. This fact confirms extrathymic origin of MG with concomitant presence of the thymoma. The same results have been confirmed in several studies observing patients with MGAT (13). The similar observation reached Masaoka et al. studying 375 patients with MG and following them for 15 years. Jaretzki reports frequent deterioration of MG after surgery due to thymoma (15). Our results show only a certain degree of improvement of MG symptomatology in patients with thymoma.

There are mentions in the most recent literature sources that the histopathological findings of follicular hyperplasia indicated favourable prognosis (16). The similar results were found after the evaluation our data. The presence of the thymic hyperplasia was found in $48 \%$ patients and the achieved complete stable remission in connection with thymic hyperplasia was $76.5 \%$. The removal of the thymic hyperplasia was expected to reduce numerically the T-cells and B-cells and doing so to diminish or completely eliminate the site of possible auto-antibodies production. The early removal avoids the development of B-cells activation in the extrathymic immunogenic tissue such as lymph nodes, spleen and bone marrow $(16,17)$.

Patients with mild forms of MG achieved better remission results. The ocular form of MG had led to better outcomes. It was shown that clinical stage I of MG predicts the most favourable outcome - spontaneous remission. Therefore the indication for thymectomy in this situation is not unequivocally accepted. On the other hand, because of the possibility of the progression to generalized form of MG, thymectomy is currently recommended.

Some studies suggested that the duration of MG could influence the effect of thymectomy Based on our results and conclusions, patients with history of illness symptoms from beginning to diagnosis up to 9 months and with the total duration of $\mathrm{MG}$ symptoms till the surgery less than 17 months had much better chance for better outcome. Above mentioned facts carry a significant benefit to patient overall prognosis.

\section{Conclusion}

Despite of the recent progresses in drug and immunological treatment strategies resulting in improved MG treatment results, the surgical treatment - thymectomy - plays an important role in the currently available complex treatment approach. However, the effectiveness of thymectomy in MG cases is still questionable, difficult to define and prove. So far, no prospective randomized studies have definitively confirmed the effectiveness and definitive role of the thymectomy in the treatment of MG. Nevertheless, the thymectomy is considered to be an effective tool in the reduction or even in complete elimination of the necessity of drug treatment. Because of early diagnosis and subsequent surgery without a delay, the thymectomy offers significantly much higher remission rate. This is desired and favourable outcome for all of us - medical personnel involved in the patient care and treatment of mysterious MG, but the most importantly, for every person in our statistic file - our patient.

\section{References}

1. Špalek P, Schnorrer M, Krajč T. Imunopatogenéza paraneoplastickej myasténie gravis asociovanej s tymómom. Neurológia 2010; 5: 7-11.

2. Špalek P. Myasténia gravis (minimonografia). Čes Slov Neurol Neurochir 2008; 71/104: 7-23.

3. Drachman DB. Therapy of myasthenia gravis. In: Engel AG. Neuromuscular junction disorders. Amsterdam; Elsevier 2008: 253-272.

4. Gold R, Hohlfeld R, Toyka K. Review: Progress in the treatment of myasthenia gravis. Ther Adv Neurol Dis 2008; 1: 99-114.

5. Kaminski HJ. Treatment of myasthenia gravis. In: Kaminski HJ. Myasthenia gravis and related disorders. New York: Humana Press, 2009: 157-174.

6. Spalek P, Schnorrer M, Sitarova K. Thymectomy in 347 myasthenia gravis patients (1990-2006). Eur J Neurol 2007; 14 (Suppl 1): 270.

7. Newsom-Davis J. The emerging diversity of neuromuscular junction disorders. Acta Myol 2007; 16: 5-10.

8. Leite MI, Jones M, Ströbel P et al. Myasthenia gravis thymus: complement vulnerability of epithelial and myoid cells, complement attack on them, and correlations with antibody status. Am J Pathol 2007; 171: 893-905.

9. Conti-Fine BM, Diethelm-Okita B, Ostlie $\mathbf{N}$ et al. Imunopathogenesis of myasthenia gravis. In: Kaminski HJ. Myasthenia gravis and related disorders. New York: Humana Press, 2009: 43-70.

10. Špalek P. Tymómy a paraneoplastická autoimunita. Čes Slov Neurol Neurochir 2002; 65/ 98: 367-373.

11. Hoch W, McConville J, Helms S et al. Autoantibodies to the receptor tyrosine kinase MuSK in patients with myasthenia gravis without acetylcholine receptor antibodies. Nat Med 2001; 7: 365-368.

12. Kondo Y, Monden Y. Thymoma and myasthenia gravis: a clinical study of 1,089 patients from Japan. Ann Thorac Surg 2005 79: 219-224.

13. Roth T, Ackermann R, Stein R et al. Thirteen years follow-up after radical transsternal thymectomy for myasthenia gravis. Do short-term results predict long-term outcome? Eur J Cardiothorac Surg 2002 4: 664-670.

14. Newsom-Davis J, Cutter G, Wolfe GI et al. Status of the tymectomy trial for non-thymomatous myasthenia gravis patients recieving prednisone. Ann N Y Acad Sci 2008 1132: 344-347.

15. Jaretzki A III, Barohn RJ, Ernstoff RM et al. Myasthenia gravis: recommendations for clinical research standards. Task Force of the Medical Scientific Advisory Board of the Myasthenia Gravis Foundation of America. Ann Thorac Surg 2000 70: 327-334.

16. Lin MW, Chang YL, Huang PM et al. Thymectomy for non-thymomatous myasthenia gravis: a comparison of surgical methods and analysis of prognostic factors. Eur J Cardiothorac Surg 2010 37:7-12.

17. Budde JM, Morris CD, Gal AA et al. Predictors of outcome in thymectomy for myasthenia gravis. Ann Thorac Surg 2001 72:197-202.

Received September 9, 2015. Accepted November 11, 2015. 